\section{Panophthalmitis in Dengue Fever}

We report a rare case of panophthalmitis in dengue fever. A 6 year old girl presented with 5 days fever and rash on the lower extremities, flushed face and myalgia. Examination revealed fever of $38^{\circ} \mathrm{C}$, erythematous rash, and flushed face. Investigations revealed leucopenia and thrombocytopenia. Dengue serology was positive (both $\operatorname{IgM}$ and $\operatorname{IgG})$. Ultrasound showed ascitis and right pleural effusion. Next morning, she complained of severe pain in the left eye. On examination, right eye was normal. Vision in the left eye was $6 / 18$. Fundus examination revealed focal leaks of exudates from venous ends of retinal vessels at superior quadrant. By evening, the left eye was swollen, with intolerable pain and vomiting. Examination revealed shallow anterior chamber, intense ciliary congestion, cloudy cornea; percluding evaluation of underlying details. Vision was reduced to perception of light. Intraocular pressure was increased. A diagnosis of acute angle-closure glaucoma was suspected and was treated accordingly. By next morning, there was gross loss of vision, proptosis and corneal clouding. Anterior chamber showed organized disc of exudates. Panophthalmitis was suspected and ultrasound was done which revealed thickening of choroid, sclera and exudation of vitreous. MRI confirmed the diagnosis and showed diffuse inflammatory thickening of the left ocular coats with hazy vitreous, peri-ocular extensions of the inflammatory process involving both pre and post septal soft tissues, retro-orbital fat and peri-optic neural sheath showed inflammatory changes.

Ocular manifestations in dengue, though rare, are not uncommon, with $20 \%$ having ocular pain [1] and $40.3 \%$ having subconjunctival hemorrhage, dilatation and tortuosity of retinal vessels and hard exudates [2]. Chorioretinitis, retinitis, retinal vasculitis and optic nerve involvement have been found to be associated with dengue [3]. Anti-IgM dengue antibody was found to be positive in $18 \%$ of patients with multifocal retinitis [4]. The triad of eye flashes, floaters and blurring of vision was highly predictive for the development of retinal hemorrhages [5]. The pathogenesis of panophthalmitis is not known. It could be the part of immunologic and inflammatory response to the dengue virus infection. The child

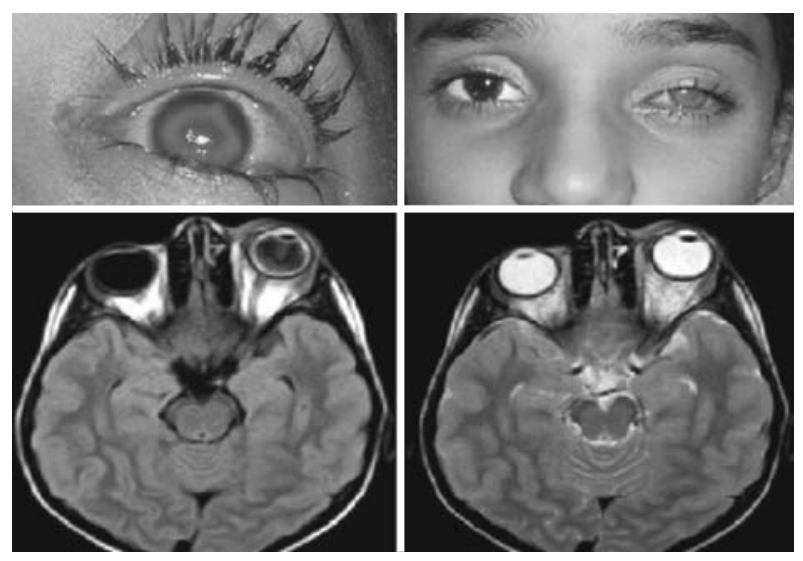

FIG.1 Showing cloudy cornea, and MRI sections of panophthalmitis.

recovered, with visual loss of left eye.

Ocular manifestations in dengue are rare, but can be as serious as panophthalmitis in this child. So a systematic ophthalmic examination in patients with dengue fever, especially with ocular symptoms, is mandatory.

Siva SaRanaPPa SB AND *HN SowbHagYa Departments of Pediatrics and *Ophthalmology, Kempegowda Institute of Medical Sciences Bangalore, Karnataka. drsharan727@gmail.com

\section{REFERENCES}

1. Humayoun MA, Waseem T, Jawa AA, Hashmi MS, Akram J. Multiple dengue serotypes and high frequency of dengue hemorrhagic fever at two tertiary care hospitals in Lahore during the 2008 dengue virus outbreak in Punjab, Pakistan. Int J Infect Dis. 2010;14:e54-9.

2. HK Kapoor, Saloni B, Mary J. Ocular manifestations of dengue fever in an East Indian epidemic. Can J Ophthalmol. 2006;41:741-6.

3. Khairallah M, Chee SP, Rathinam SR, Attia S, Nadella V. Novel infectious agents causing uveitis. Int Ophthalmol. 2010;30:465-83.

4. Shukla J, Saxena D, Rathinam S, Lalitha P, Joseph CR, Sharma S, et al. Molecular detection and characterization of West Nile virus associated with multifocal retinitis in patients from southern India. Int J Infect Dis. 2012; 16:e53-9.

5. Seet RC, Quek Am, Lim EC. Symptoms and Risk Factors of ocular complications, following Dengue Infection. J Clin Virol. 2007;38:101-5. 